$M-\bar{R}$, no point of $R^{\prime}$ is a point of $H$. Therefore every point of $R^{\prime}$ is an $M$-boundary point of $H$, that is, $R^{\prime}$ is a subset of $H^{\prime}$. Hence $R^{\prime} \equiv H^{\prime}$.

The University of Pennsylvania

\title{
A THEOREM ON CONNECTED POINT SETS
}

BY C. KURATOWSKI AND C. ZARANKIEWICZ

1. Introduction. The purpose of this paper is to prove the following theorem.

Theorem. If $S$ is a connected point set and $Z$ is the set of all points such that $S-p$ is neither connected nor the sum of two connected sets, then $Z$ is finite or countable.

2. Lemma. If $S, P$, and $Q$ are three non-vacuous connected sets (or points), and if

$$
\begin{aligned}
& P+Q \subset S, \\
& A \subset S-P, \\
& A \cdot Q=0,
\end{aligned}
$$

$$
\begin{aligned}
& P \cdot Q=0, \\
& B \subset S-Q, \\
& B \cdot P=0,
\end{aligned}
$$

(7) $A$ and $S-P-A$ are mutually separated,

(8) $B$ and $S-Q-B$ are mutually separated, then $A \cdot B=0$.

Proof. By (1) and (3), $A+P \subset S$. Hence, by (2) and (5), $A+P=(A+P) \cdot(S-Q)$. By (4), $S-Q=B+(S-Q-B)$. Therefore

$$
A+P=(A+P) \cdot B+(A+P) \cdot(S-Q-B) .
$$

It follows from (2) and (6) that $P=P-Q-B \subset(A+P)$ $(S-Q-B)$. Since $P \neq 0$, we have

$$
(A+P) \cdot(S-Q-B) \neq 0 .
$$

Now, by (8), the sets $(A+P) \cdot B$ and $(A+P) \cdot(S-Q-B)$ are mutually separated. On the other hand, by virtue of a 
theorem of Knaster and Kuratowski, ${ }^{*}$ and by (3) and (7), the set $A+P$ is connected. Hence, by (9) and (10), $(A+P)$ - $B=0$, that is, $A \cdot B=0$.

3. Proof of the Theorem. Let

$$
R_{1}, R_{2}, \cdots, R_{n}, \cdots
$$

be the sequence of all circles (spheres) which are such that their radii and the coordinates of the center are rational; and let

$$
(1,2,3),(1,2,4), \cdots,(i, j, k), \cdots
$$

be the sequence of all systems composed of three different positive integers.

We shall first show that, for every point $p$ of $Z$, there exist three sets $A, C$, and $D$ and a system of indices $(i, j, k)$ such that

$$
\begin{aligned}
& A, C, \text { and } D \text { are mutually separated, } \\
& \qquad \begin{array}{c}
S-p=A+C+D, \\
S \cdot R_{i}=A \cdot R_{i} \neq 0, \\
S \cdot R_{j}=C \cdot R_{j} \neq 0 \neq D \cdot R_{k}=S \cdot R_{k} .
\end{array}
\end{aligned}
$$

By hypothesis, $S-p$ is not connected. Hence $S-p$ may be decomposed into two mutually separated sets; one of these two sets can itself be decomposed into two mutually separated sets, since by hypothesis $S-p$ is not the sum of two connected sets. It follows that there exists at least one decomposition of $S-p$ into three non-vacuous mutually separated sets $A, C$, and $D$. Let $a, c$, and $d$ be three points in $A, C$, and $D$, respectively. It is obvious that the point $a$ may be surrounded by a circle $R_{i}$ which contains no point of $C+D+p$. Similarly $c$ and $d$ belong to two circles $R_{j}$ and $R_{k}$ that contain no point of $A+D+p$ and of $A+C+p$, respectively. Therefore the conditions (15) and (16) are fulfilled.

* Sur les ensembles connexes, Fundamenta Mathematicae, vol. 2 (1921), p. 210 , Theorem VI. 
Thus we have shown the existence of a system $(i, j, k)$ having the property that there exists at least one system of three sets $(A, C, D)$ satisfying the formulas (13)-(16). We denote by $\sigma(p)$ the first system in the sequence (12) having that property.

We shall prove that, if

$$
p \neq q
$$

then $\sigma(p) \neq \sigma(q)$.

Suppose, on the contrary, that $\sigma(p)=\sigma(q)=(i, j, k)$. Hence there exist three sets $A, C, D$ satisfying the conditions (13)-(16) and three sets $B, E, F$ such that

$$
B, E \text {, and } F \text { are mutually separated, }
$$

$$
\begin{gathered}
S-q=B+E+F, \\
S \cdot R_{i}=B \cdot R_{i} \neq 0, \\
S \cdot R_{j}=E \cdot R_{j} \neq 0 \neq F \cdot R_{k}=S \cdot R_{k} .
\end{gathered}
$$

From (15) and (20) it follows that $A \cdot R_{i}=B \cdot R_{i} \neq 0$. Hence

$$
A \cdot B \neq 0
$$

and similarly, $C \cdot E \neq 0 \neq D \cdot F$.

By (17), (14) and (13) $q$ belongs to one and only one of the sets $A, C$, and $D$. Similarly, $p$ belongs to one and only one of the sets $B, E$, and $F$. Clearly we may suppose that neither $q$ belongs to $A$ nor $p$ to $B$, so that

$$
A \cdot q=0=B \cdot p .
$$

Now, by (13) and (14), $C+D=S-p-A$. It follows by (13) that $A$ and $S-p-A$ are mutually separated. Similarly, $B$ and $S-q-B$ are mutually separated. Therefore, by virtue of the Lemma and by (17), (14), (19), and (23) we have $A \cdot B=0$. But this is a contradiction of (22).

Thus we have shown that to different points of the set $Z$ correspond different systems composed of three positive integers. Hence the set $Z$ is countable. 
4. Remarks. A. It is to be noted that the above proof was carried out without the aid of the Zermelo Axiom* and that it gives a mode of enumerating the points of $Z$. That is, $Z$ is effectively countable (in the sense of Borel-Sierpinski).

B. By means of a remark of B. Knaster, the argument used above may be applied to prove the following more, general theorem. If $S$ is a connected set and $Z$ is a class of mutually exclusive, connected, and relatively (to $S$ ) closed sets $X$, such that $S-X$ is neither connected nor the sum of two connected sets, then the class $Z$ is countable.

C. In case $S$ is an acyclic continuous curve (i. e., a continuous curve that contains no simple closed curves), then the points of the set $Z$ are identical with points of ramification of $S . \dagger$ Thus, our Theorem has as a consequence the theorem of Wazewski-Menger $\neq$ to the effect that the set of points of ramification of a tree is countable.

* The Zermelo Axiom was used by Wazewski (Annales de la Société Polonaise de Mathématique, vol. 2 (1923), p. 169) to prove a particular case of our Theorem, namely the case $S$ is a continuous curve.

By a method analogous to that used above, the following theorem that was proved with the aid of the Zermelo Axiom by R. L. Moore in a less general form (see Proceedings of the National Academy of Sciences, vol. 9 (1923), p. 102), and by C. Zarankiewicz (Fundamenta Mathematicae, vol. 9 (1927), p. 140, Theorem 9) may be proved without having reference to that axiom:

If $T$ is a connected subset of a connected set $S$ and $W$ is the set of points each of which belongs to $T$ and disconnects $S$ but not $T$, then $W$ is countable.

For, suppose $p$ is a point of $W$. Then there exists a circle $R$ having the property that there exists a set $A$ such that (i) $A$ and $A-p-S$ are mutually separated, (ii) $A \subset S-T$, (iii) $S \cdot R=A \cdot R \neq 0$.

Let $n(p)$ denote the lowest index such that the circle $R_{n(p)}$ of the sequence (11) has that property. Now, if $p \neq q$ then $n(p) \neq n(q)$. Suppose, on the contrary, that $n(p)=n(q)$. Hence there exists a set $B$ such that (iv) $B$ and $S-q-B$ are mutually separated, (v) $B \subset S-T$, (vi) $S \cdot R=B \cdot R \neq 0$. It follows, by (ii), that $A \cdot q=0$ and, by (v), that $B \cdot p=0$. Therefore, by (i), (iv), and the Lemma, we have $A \cdot B=0$. But this is a contradiction of (iii) and (vi). Thus the set $W$ is effectively countable.

† See K. Menger, Ueber reguläre Baumkurven, Mathematische Annalen, vol. 96 (1926), p. 574. A point $p$ is said to be a point of ramification of a (regular) curve if the curve contains three $\operatorname{arcs} L_{1}, L_{2}, L_{3}$ such that $p=$ $L_{1} \cdot L_{2}=L_{1} \cdot L_{3}=L_{2} \cdot L_{3}$.

‡ Wazewski, loc. cit., p. 169, Menger, loc. cit., p. 576. 
This theorem may be generalized as follows. Suppose $S$ is a acyclic continuous curve locally at each of its points (i. e., for each point $p$ of $S$ there exists an acyclic continuous curve $T_{p}$ contained in $S$ and such that $p$ is not a limit point of $S-T_{p}$ ). It follows, by the Borel covering theorem, that the set of all points of ramification of $S$ is still countable.

A direct consequence of this statement is the following result* obtained by Alexandroff: If $S$ is a continuous curve of finite connectivity then the set of all points of ramification of $S$ is countable. For, $\ddagger$ a continuous curve of finite connectivity is a tree locally at each of its points.

Now, if $S$ is a plane non-dense continuous curve that disconnects the plane in a finite number of domains, then $\S S$ has a finite connectivity. Thus we may state the following result. The set of points of ramification of a plane non-dense continuous curve that disconnects the plane in a finite number of domains is countable.\|

UNIVERSITY OF WARSA $\mathrm{W}$

* P. Alexandroff, Ueber kombinatorische Eigenschaften allgemeiner Kurven, Mathematische Annalen, vol. 96 (1926), p. 552, Corollary 4.

† A (one-dimensional bounded) continuous curve is said to be of finite connectivity if it contains at most a finite number of simple closed curves. This definition is equivalent to that given by Alexandroff, loc. cit., p. 541, by virtue of the Lemma 15 of the mentioned paper of Zarankiewicz.

$\ddagger$ See Menger, loc. cit., p. 574.

$\S$ See Alexandroff, loc. cit., p. 527.

\| This statement does not remain true if the hypothesis that the number of complementary domains is finite be omitted, as is seen on an example of a continuous (regular) curve composed exclusively of points of ramification, given by W. Sierpinski, Comptes Rendus, vol. 160 (1915), p. 305; see also this Bulletin, vol. 33 (1927), p. 106. 